\title{
The Tool That Has to Build Itself: The Case of Dutch Geo-Data
}

\author{
Walter T. de Vries ${ }^{1, *}$ and Gianluca Miscione ${ }^{2}$ \\ ${ }^{1}$ University Twente, Enschede, Netherlands \\ w.t.devries@utwente.nl \\ ${ }^{2}$ University College Dublin, Ireland \\ gianluca.miscione@ucd.ie
}

\begin{abstract}
Standardisation is one of tools of Electronic Government (EGov). It refers to the development where individuals and organizations develop and/or adhere to standard IT solutions and associated work processes. Studies on standardization in information technology (IT) emphasize either only the technical side of standard construction (the 'what' and 'how'), or the socio-organizational side of the contextual processes in which standards emerge (the 'who' and 'when'). Our article has an alternative, socio-technical, approach, which emphasizes 'where' standards crystallize. Our empirical field to find where crystallization occurs concerns the geoIT sector. Through a qualitative approach, the data show that standard crystallization occurs at the hubs of inter-organizational relations, rather than at the top or the bottom of formal organizations. This claim is important because it contradicts the common strategy of standardization, which is largely centralized. Even though government has centralized the creation and distribution of geoIT standards, their actual creation and crystallization occurs at a more decentralized level: across municipalities. The conclusion is that bringing the standardization discussion to a point of where standardization actually happens, provides a better understanding of the socio-technical dynamic of governance of inter-organizational IT.
\end{abstract}

\section{Introduction}

When designed and implemented within an e-government framework, information technologies (IT) are intended to be both technologies for the bureaucracy -because it is in this kind of organizations that they are implemented - and technologies of the bureaucracy, as they aim at extending public administration functioning in relation to citizens. Either way, the IT is conceived as a tool in the hands of decision makers. Such a view on technology, usually contrasted to a techno-deterministic view, falls short in accounting for the quite frequently observed 'drift' [1] of large information systems. Although we would not make a groundbreaking claim that evolution of technology often eludes designers' intentions, here we adopt a stance which resonates more with [2], who conceptualize technology as 'gestell' (i.e. enframing) rather than a

\footnotetext{
* Corresponding author.
} 
malleable tool at disposal of will. Our case corroborates their phenomenological stance, and expands it by identifying where crucial developments of geoIT actually happen. More precisely, we focus on the two-way relation between geoIT and interorganizational processes.

Harmonizing technical with socio-organizational analytical perspectives with the aim to construct a comprehensive perspective on IT development and standardization has never been immediate. Specifically, engineering is oriented to define what works [3], so functionality is the legitimizing source for this kind of research. Contrastingly, socio-organizational science approaches are closer to the traditional science epistemology, aimed at explaining and predicting rather than doing. In the latter case, explanatory power -aimed at pushing the boundaries of what is known- legitimizes research. The mismatch between how the two communities "make a point" make it difficult to combine their views, therefore to develop a consistent conceptualization of e-government.

Given this mismatch this article proposes a different viewpoint, empirically first. Instead of emphasizing the construction of governance tools, the technical standards, and instead of emphasizing the contextual conditions for governance, the socioorganizational environment, we emphasize 'where' agency performs action, 'where' it takes place. In particular, we focus on 'where' standards are consolidated. We assume that analyzing the areas of influence in dynamic inter-organizational relations addresses this 'where' question. Our empirical domain is the Dutch geoIT sector. This sector, made up of professionals and scientists using and developing ICT related to (geo-)spatial processes and phenomena, is a sector where dynamic interorganizational relations have developed historically. Such relations can be framed by the concept of Spatial Data Infrastructures (SDIs). SDIs are the socio-technical networks of 'geospatial technologies and institutional arrangements and practices that allow for the disclosure and sharing of geospatial data among various levels of government' [4]. Over time, SDIs have created standards of inter-organizational activities. Yet, whose standards are adopted, and where such standards emerge is largely unknown. Therefore, in this paper we look for where SDI standardization happens. We frame this question as a question of SDI crystallization (echoing the snowflakes from Staring and Titlestad [5], which gives primacy to so called 'de facto' standards.

This article continues in section 2 with an explanation of what the concept of information infrastructure (of which SDIs are an instance) crystallization would entail, and why it would be useful as an alternative stand to study standardization as a governance mechanism of inter-organizational IT. This section also presents how the dimensions of information infrastructure crystallization would apply to SDI developments, and how this would translate into empirical questions. Section 3 describes which cases are representative for SDI development, and for addressing the main research questions. Section 4 presents the results of the data collection. The concluding section derives how the findings provide added value to the knowledge of Egov tools. 


\section{Two Views on Where and How geoIT Standards Develop}

Information infrastructures depend, in a way or another, on standards. Studies on standards either emphasize the technical side of standard construction i.e. the 'what' and 'how' [6-8], or the socio-organizational side of the contextual processes in which standards emerge, i.e. the 'who' and 'when' [9-11]. Where technical studies tend to emphasize the construction of standards as a solution to technical interoperability, the socio-organizational studies emphasize both the regulatory nature of standards within organizations, and the patterns of power preceding or resulting from standard-making.

Standards relate however also to scale, i.e. the 'where', either as a cause or an effect. In current discussions on how standards are formed and what role standards play in this formation of SDIs, two opposite agency standpoints are visible - through centralized and through decentralized agency (agency in its broad meaning of what performs action). When agency is argued as centralized, it can be localized more easily. When agency tends to be distributed, it can be situated in an "area" (corresponding to interest groups, lobbyists, organizations, etc.). Therefore, understanding the distribution of action across actors helps us in identifying the locus of SDI crystallization.

The centralized agency view posits that geoIT standards originate from national statutory tasks and centrally led initiatives. Public sector actors are linked through a pyramid of different levels of public administration [12]. The statutory mapping tasks of national actors require them to decide on national mapping standards. The only way for national agencies to have interoperable maps within all government agencies is through requiring other agencies at other administrative levels to use the same mapping standards. In this view the highest level SDI is the aggregate of all lower level SDI. The highest level standards are the aggregate of all lower level standards. This pyramid view assumes undisputed lines of power, which are visible through the formalization of relations between the actors through bilateral or multilateral procedures, laws and/or regulation; the working processes follow agreed rules, laws, written and distributed regulations. The decisions on who is in the SDI and who is out are designed to be formal, and the standards are pre-defined and distributed along the formal lines.

In contrast the decentralized agency view posits that geoIT standards are shaped decentrally. At the extreme, there is a relevant role of voluntary production of geoinformation through crowdsourcing initiatives, and by citizens. These people drive the change in technology and in doing so implicitly set the standards of data, data sharing and exchange, and work processes of data collection and distribution. In more recent years, "voluntary geo-information" (VGI), is challenging the exclusivity of geoIT handling by public organizations [13-15]. The decentralized process of standards development is one whereby actors are assumed to operate within a large open network of relations. There is no clear-cut boundary between who is in and who is out. Recent VGI efforts and standard development operates similarly to an "internet-like" process, the standardization process co-develops along with globally dispersed activities. One of the questions is thus: who or what decides on the rules "here", in this setting. The relations are mostly built upon informal links between actors and stakeholders, and what drives these relations is a common, yet temporary, interest. 
Comparing both views shows a difference in locus of standards crystallization. The first view emphasizes the coercive nature of national and central governance mechanisms. Standards emerge in this view as a direct effect of legislation and regulation. Contrastingly, the second view emphasizes emergence of standards at local levels. Standards in this view are caused though the autonomy of local actors, whose aggregated actions constitute certain decisions and practices. Although both views acknowledge that the discontinuation of one standard and the start of a new standard is more gradual than punctuated, in both perspectives standards change over time, and the origin of this change relates to the context in which actors operate. It is therefore remarkable that neither of the two views recognizes that professional actors often operate through professional networks which are loosely connected to the national or local level [16, 17].

Information infrastructure studies focus on the relations between information systems, and the underlying processes between actors working with the systems once information systems are connected. An information infrastructure perspective could address this void. From this perspective, inter-organizational relationships depend on which agency can exercise what type of influence on organizations and individuals. Standards, in this view, have the effect of regulatory agency beyond a single organization, or a single level [18]. Hence, standards (or lack of them) do not emerge within single organizations, but emerge and crystallize in inter-organizational processes regardless of the level at which they take place. By emphasizing the network relations between actors at any level, this study does not emphasize the self-contained organizational structure so much, but instead the organizing processes [19]. Understanding the organizing processes of standardization thus implies having to look into the diverse relations across organizations. The characteristics of the relations thus reveal the location of where standards form and where and how standards crystallize.

\section{Methodology and Cases}

Our overarching empirical research question is: Where do inter-organizational relations working with geoIT produce SDI standards? This has three main components which were each evaluated: 1) the cases of inter-organizational relations in connection to the use of geoIT; 2) the artifacts that justify that SDI standards are being crystallized; 3) the determination of the location of the production / emergence of standards.

Addressing the first component relied on case study methodology. This is relevant, because the boundaries of the phenomenon under consideration, the locus of standardization, and the context, the geoIT world of practitioners, and the public administrative structures, were unclear from the onset. As [20] argue "the structure of an organizational field cannot be determined a priori, but must be defined on the basis of empirical investigation" (p.65). The review and comparison of cases in the Netherlands drew upon extensive experience and subsequent empirical data collections in the area by de Vries [21-23]. The selection of cases for this study relied on one element which 
was different between cases: the control structure. This allowed verifying if the location of regulatory control was relevant in coordinating of the inter-organizational relation. Using this selection criterion derived three cases: Cadastral case, Dataland and Dimpact.

The Cadastral case refers to a national organization 'Kadaster' (the National agency for Land administration and land registration) with sub-national working relations with municipalities. The Kadaster historically has strongly coordinated the interorganizational work processes to maintain parcel-based spatial information, captured with geoIT. The Dataland case refers to a national association Dataland made up by municipalities. A central office Dataland centrally aggregates real estate information through coordinating work processes in municipalities. Real estate information is collected and distributed through geoIT. The Dimpact case refers to an association of various municipalities. The Dimpact office coordinates ICT work processes for municipalities. This office relies on staff from member municipalities in the development of information and ICT solutions for other municipalities. GeoIT is one of the components.

Our conceptual entry point for the second component is the hypothesis that either type of agency (central or peripheral) creates SDI crystallization. We assume hereby that such crystallization is visible along three dimensions, following Miscione, Staring and Georgiadou [24]. These include accreditation, coalition, alongside with the activities related to the adoption of standards. Accreditation refers to the influence of actors to guarantee access. Coalition refers to the possibilities of establishing couplings between data and related activities and organizations. Adoption of standards refers to both the data and to organizational processes compliance to common guidelines. These aspects do not distinguish technical and socio-organizational dimensions a priori. They are also mutually dependent, and help in describing interorganizational processes. For example, a public body may decide what standards have to be used for geo-information. Nevertheless, existing systems in use in accredited organizations may require different strategies of data integration. The method of collecting data about the values within these three dimensions was a combination of semi-structured interviews, complemented by a workshop with representatives from municipalities, and a survey among geoIT practitioners. We conducted 15 in-depth interviews with representatives within all cases. We transcribed all interviews and the discussions during the workshop. The survey data were collected through an online questionnaire. We coded both the transcriptions and all other relevant documents with the help of a qualitative analysis support software. All data were coded applying an open coding strategy of Lee [25] and Lewins [26]. In total there were 99 respondents.

For the third component we relied on an interpretative approach. Upon completion of the coding we specifically queried both 'change' and 'location' aspects emerging from the coding results. We assumed hereby that agency can be seen through respondents' references or associations to both change and to the origin of change. In both cases the interpretation of the coding and associated quotations relied on both explicit and implicit references to 'change' and 'origin of change'. 


\section{$4 \quad$ Findings}

We present our findings following the three specific research questions.

\section{Cooperation using geoIT}

In each case the actors shared responsibilities in relation to the geoIT data. With regards to the data this concerned the harmonization of georeferencing definitions (such as coordinates and zip codes), the type of spatial objects to use (collection of lines, areas, or points), the type of attributes to include per spatial object and the scale and associated required accuracy of data. The type of data varied per case. In the Cadastral case the Kadaster maintained collections of parcels, each having parcel identifiers, whereas each of the municipalities maintained collections of buildings, each having address identifiers. Moreover, The Kadaster and Dataland organizations maintained their own database on their own server, whereas the Dimpact organization relied on the generation of services extracting from the data collections within each municipality.

The crucial agreements on procedures concerned decisions on data collection and maintenance. Municipalities are by law responsible for the definition and the collection of buildings and address data, and are implicitly responsible for the quality (accuracy, reliability) of these data. The Cadastre on the other hand maintains these data for all municipalities. Dataland coordinates the maintenance of cross-boundary municipal data on behalf of the municipalities. Not entirely surprisingly, the influence that the central office in each case (The Headquarter Cadastre, and the Bureaus of Dataland and Dimpact) can exercise on local municipalities diminishes as the central office is further away (both physically and institutionally) from these municipalities. A respondent from a smaller municipality stated ironically on the data collection and distribution responsibilities: It is all very nice what the central office comes up with, but we don't need them. You only hear once, and afterwards you really have to push to get any information from them. It is all written for bigger municipalities. It is absolutely unusable stuff for smaller and medium sized municipalities like us.

Sharing data to third parties was another joint concern, working with the huge amounts of data on a daily basis is very different among the cases. A Kadaster representative stated We just have to process as many transactions as possible. 500,000 transfers of deeds, 500,000 transfers of mortgages, 20 million digital transactions. It's just production work. A Dataland representative highlighted: In our regular database we have 7 million objects, yet if no one wants to use any of those data it would be dead capital. Still, we receive all our data from the municipalities, and from all the revenue that we generate, $15 \%$ goes back to the municipalities to pay for all their delivery costs. From the remaining $85 \%$ we can pay our own costs of data management. In other words, both organizations work as data factories, with factory-like business models and business attitudes, and the procedures therefore emphasize immediate efficiency interests. In both Dataland and Dimpact the municipalities agree jointly, via the coordination bureaus, on the conditions of how and to whom to distribute their individual or combined datasets. Since the Cadastre, Dataland and individual municipalities own fees through the distribution of data, customers are crucial for 
the existence of the organizations. A Dataland representative even stated during an interview: Our customer focus is so big... It is our lifeblood.

So, we can note a geographical and topical convergence between these organizations. Both cooperation and standards are created and sustained by actors which are mostly geographically clustered and have ongoing relations already. Dimpact members are often geographically clustered groups of municipalities, and Dataland nonmembers are also geographically clustered. This does not mean that data quality is homogeneous within clusters, because as for example within Dataland it is noted that: Almere and Lelystad [both Dataland members] are adjacent cities. They have the same history, both were created in Flevoland province. Than one would expect a similar quality, but they score very different in quality indicators. While membership may be geographically fostered, data quality standard within the organization is also related to local information management practices. Our explanation is that a clustering of actors follows a narrow set of thematic data applications, such as real estate (Cadastral and Dataland cases).

2. Shaping of inter-organizational relations in terms of accreditation, coalition and adoption of standards

The accreditation in the cadastral case mainly depended on the degree to which the Kadaster was able to convince the ministry of spatial planning of their price policies. The Kadaster could autonomously decide on all data management processes, yet only needed a ministerial approval for the pricing policy when distributing data. The Kadaster thus implicitly accredited the data quality, yet the price for the data required an accreditation from the ministry. In the Dataland case, the Dataland head office set out a series of data quality parameters, which they did not enforce, yet which they monitored within municipalities. The purpose of the monitoring was to develop gradually a list of best practices, and a list of best municipalities adhering to these practices. The Dataland head office accredited data quality through organizing a price for the best municipality. The accreditation thus relied on the promotion of this price by peers, and the positive image for municipalities attached to this price. In the Dimpact case, the setting of the data and services quality relied heavily the technical representatives of individual municipalities. They jointly decided on certain quality parameters, such as specific GIS solutions, and were though these decisions implicitly accrediting themselves.

The organization of coalitions differs per case. The Kadaster was maintaining individual contracts with each municipality, yet this would change soon given the implementation of national key registers. New legislation on key registers would distribute data responsibilities for collection and distribution to the Kadaster and municipalities in more detail. This new legislation is likely to change the contractual agreements between the organizations. In the Dataland case the coalition relied on inter-related structures of memberships. The Dataland central Bureau administers memberships, and coordinates cohesion among members. Dataland itself is a cooperative of municipalities with Dataland foundation and Dataland corporation, and an executive Bureau Dataland. In the Dimpact case, the coalition was organized voluntary membership rules. Municipalities were voluntary members to an association of municipalities, 
while an executive bureau Dimpact coordinated the coalition alignment at technical and policy level.

With regards to the adoption of standards in the Cadastral case, all data and operational processes were largely set by the Kadaster, operating under the authority of a Cadastral law. In the implementation of this law the central theme was how to generate 'efficient core registration (of government data)'. In the Dataland case, The Dataland Bureau was setting validation rules for checking the data quality, and applied these rules to check data of individual municipalities. In this process the core theme was 'improving accessibility (to government data)'. In the Dimpact case, the technical solutions for data problems were created by private companies in outsourcing contracts. Central in the management of the data standard and data production standard creation was the repeated catch phrase 'improving (e-) service to citizens'. These 'catch phrases' mentioned during individual interviews are exemplary of the how actors tried to steer standards in a particular direction: We do not want to be politically interesting, but we lost 40 or 50 million revenue due to the real estate crisis. As a result we decreased out cost with 15 million. The mechanism that we agreed was to operate like a normal business. (Senior Kadaster representative); Our whole story is about access to government information. 'That is the core and mission of Dataland. That is the point. Dataland wants to make public municipal data more accessible'. (Senior Dataland representative); Dimpact is indeed a cooperation between municipalities, which, just like any other association, become a member to a central image, that of e-service. Like you join a tennis club to play tennis, you join our club to realize e-services as member. (Senior Dimpact representative)

The Kadaster representative has a clear preference for standards based on (cost) efficiency norms. The Dataland representative emphasizes accessibility as the most crucial norm. The Dimpact representative emphasizes collectivity as a key norm. The efficiency of the Cadastral case is quite different than the improving accessibility, or improving e-services to citizens. Which standards emerge as a result is however greatly affected by these 'buzz words'. Despite the fact that coordination of standards relied on centralized coordination activities, the results under 'responsibility' show that the space of influence from the central office decreases, as the distance to where standards are actually used (at the level of municipalities) decreases. A possible explanation is that the acceptance of centralized standards relates to the degree by which municipal staff feels themselves represented in the decisions on standards. In the Cadastre case, a number of representatives of larger municipalities explicitly rejected to accept the central standards, and preferred to use standard resulting from a process in which they were involved themselves. The argument was that internal alignment of geoIT standards with other internal departments received higher priority than alignment with external agencies. Smaller and medium sized municipalities preferred to be included in clusters of municipalities, so that they could follow and influence geoIT developments more directly. Both Dataland and Dimpact provided this facility, as their central office was directly constituted by the municipalities themselves (Dataland), or was directly implemented by municipal staff (Dimpact). 


\section{Where do changes occur?}

Two types of changes were apparent. The first type reflected a gradual change. The size of Dataland and Dimpact cooperation consortia gradually increased even during the data collection process related to these cases. The number of actors involved increased both in number of organizations and in number of professionals involved. The staff members working in these cases indicated that this increase of actors made the coordination of standards, even if it were on a voluntary basis, more complex. Despite this increase of members there was no change in the coordination and governance structures between the organizations. This is remarkable, because one would expect the work processes and the way that people agree on these work processes might get more complicated, and hence might need adaption.

A second type of change was more punctuated. The introduction of the new law on base registrations affected all actors in all cases. Although the new law had been in preparation for a considerable amount of time, and although many of the technical staff members had been involved in proposing the details of this law through working groups, still the approval of the law had immediate legislative consequences. As a result, staff members perceived a change in their management activities, in particular within the municipalities. As stated above, in the Cadastral case the distribution of responsibilities between the Cadastre organization and the municipalities changed, and had given municipalities more responsibility in the maintenance of address and real estate data. Many municipal staff members perceived however that they only had limited capacity to deal with this task, while maintaining other tasks at the same time. This additional work load required them to rethink their operational procedures, and allocation of tasks to staff.

A third type of change in the use of geoIT standards was expected, yet did not occur. The standards of the citizens (customers of the products and services of all the cases), such as open geospatial data standards, were hardly taken up within the internal processes of the cases. Some municipalities indicated adhering to open data standards principles, but neither in the statutory duties or in their voluntary agreements did they insist on relying on these open data standards. Standard geo-data handling and provision thus still strongly relies on existing working relations within the larger agencies.

In sum, one may conclude that the stability of the internal coordination structures (aiming for standards in geoIT) is not really rooted in the specific number of members in a consortia, or in either hierarchical relations (coerced by law) or in bottom-up relations (emerging from daily interaction with citizens/customers) but in the similarity of relations that the new actors had in comparison to the existing members. Coordinating standards on geoIT seems thus strongly dependent on existing coordination structures on other issues. Hence, the locus of standards crystallization is not central or peripheral, but at the point where long-term mutual relations already exist. 


\section{Discussion}

Overall, the data show the importance of a middle level ground between central administration and a famous Internet motto: "rough consensus and running code" as the way an information infrastructure establishes itself. This can also be read as a liminary position between expert and lay knowledge. Our underneath interest to focus on the 'where' question was to avoid usual accounts, deeply entrenched in the public administration -and usually elicited by interviews: "How does standardization happen?" is likely to get answers like "a formal process has been put in place, followed, different viewpoints have been considered, together with technical viability". In the cases understanding 'where' standardization of geoIT takes place cannot be reduced to understanding how SDI has evolved within the traditional public sector only. A number of relevant and emerging phenomena on the fringes of mapping agencies' core business have emerged as well. So, our research focused on the crystallization of relations across inter-organizational relations across the Dutch geoIT organizational field. We saw that geoIT standardization takes place in an environment which consists of both hard and formal relations (contracts between public authorities, with public mandates in the field of geoIT) and soft an informal ones (voluntary associations having voluntary working relations and rather loose decision and control mechanisms). Hence municipalities proved to be central middle ground between central bureaucracy and citizens, are places where standardized practices crystallize, thus where standardization occurs. It is relevant, because municipalities are non-specialized bodies for geoIT standardization. This counteracts a common assumption, as specialized bodies at national level are the primary knowledge holders of the domain. Yet, in their need to combine all sorts of top-down requirements to implement the execution of legislation on the one side, and deal with external geoIT users on the other side, actors at the municipal level act as mediators between those requirements, and de facto act as standardizors. We explain the role of municipalities by being entangled with both bureaucracy the citizens, not only through work relations, but also through representation and legitimacy.

So, we identified a significant window of opportunity at the municipal level, which is strategically between central administrations and citizens. In information infrastructure terms, the municipalities are the hubs of an emerging network, and the hubs of the network actually drive the process of crystallizing socio-technical relations into standards, rather than adopting what has been designed elsewhere. Where the traditional view would predict that there is a central definition of standard, which is then applied throughout the hubs, the findings show that hubs are at the encounter of different geo-information flows and combine them in use, this crystallizing the standards. So, as detailed below, rather than being entitled to set standards, what affect and explain standardization are the loci of accreditation and coalition. This is particularly relevant within the public sector, where standardization is expected to be led by professionalized, dedicated bodies. Our analysis identified an interesting tension between the trend towards more autonomous cooperation among smaller public organizations and organizations which are lower in the administrative hierarchy and less specialized. So, what is the role of the people at these levels, and the standards they 
are shaping? Are these people being ruled or are they ruling? Our point from the empirical cases and information infrastructure research is that this dichotomy is not very suitable to frame the problem and answer the question. Rather, we put at the center the locus where standards actually happen to crystallize. Indeed, municipalities are a crucial point of encounter along the tensions they are intertwined with, and this position sheds new light on geoIT standardization. From this perspective, 'street level' public sector officers are gatekeepers, being at the intersection of geo-information production and use. This is our proposal for a conceptualization of SDI as a tool which has a sensible degree of autonomy from what decision makers have in mind and express through designers. Such autonomy explains how and why this 'tool' also builds itself more than conventional accounts acknowledge.

\section{References}

1. Ciborra, C., Braa, K., Cordella, A.: From control to drift: the dynamics of global information infrastructures, vol. 264. Oxford University Press, Oxford (2000)

2. Ciborra, C.U., Hanseth, O.: From tool to Gestell. Agendas for managing the information infrastructure. Information Technology and People 11(4), 305-327 (1998)

3. Orlikowski, W.J.: Using technology and constituting structures: A practice lens for studying technology in organizations. Organization Science 11(4), 404-428 (2000)

4. Homburg, V., Georgiadou, Y.: A Tale of Two Trajectories: How Spatial Data Infrastructures Travel in Time and Space. The Information Society: An International Journal 25(5), 303-314 (2009)

5. Staring, K., Titlestad, O.H.: Development as a Free Software: Extending Commons Based Peer Production to the South. In: ICIS 2008 Proceedings, Paper 50 (2008)

6. Lehr, W.: Standardization: Understanding the process. Journal of the American Society for Information Science 43(8), 550-555 (1992)

7. Albrecht, J.: Geospatial information standards. A comparative study of approaches in the standardisation of geospatial information. Computers \& Geosciences 25(1), 9-24 (1999)

8. Schuurman, N.: Social perspectives on semantic interoperability: Constraints on geographical knowledge from a data perspective. Cartographica 40(4), 47-61 (2005)

9. Foray, D.: Users, standards and the economics of coalitions and committees. Information Economics and Policy 6(3-4), 269-293 (1994)

10. Hanseth, O., et al.: Reflexive standardization: Side effects and complexity in standard making. MIS Quarterly 30(special issue), 19 (2006)

11. Markus, L., et al.: Industry-wide information systems standardization as collective action: The case of the US residential mortgage industry. MIS Quarterly 30(special issue), 439465 (2006)

12. Williamson, I.P., Rajabifard, A., Feeney, M.-E.: Developing Spatial Data Infrastructures: From Concept to Reality, 352 p. Taylor \& Francis (2003)

13. Goodchild, M.F.: Citizens as sensors: the world of volunteered geography. Geo Journal 69(4), 211-221 (2007)

14. Goodchild, M.F.: Citizens as voluntary sensors: spatial data infrastructure in the world of Web 2.0. International Journal of Spatial Data Infrastructures Research 2, 24-32 (2007)

15. Coleman, D.J., Georgiadou, Y., Labonte, J.: Volunteered geographic information: The nature and motivation of produsers. International Journal of Spatial Data Infrastructures Research 4, 332-358 (2009) 
16. de Vries, W.T., Miscione, G.: Relationality in geo - information value: price as product of socio - technical networks. International Journal of Spatial Data Infrastructures Research: IJSDIR 5 (2010)

17. De Paoli, S., Miscione, G.: Relationality in geoIT software development: How data structures and organization perform together. Computers, Environment and Urban Systems (in press, Corrected Proof)

18. Brunsson, N., Jacobsson, B.: A world of standards. Oxford University Press (2000)

19. Czarniawska, B.: A theory of organizing, vol. 153. Edward Elgar (2008)

20. Powell, P.J., DiMaggio, W.W.: The new institutionalism in organizational analysis. The University of Chicago Press (1991)

21. de Vries, W.T.: Coördinaten en coördinatie: een analyse van overheidssamenwerken met geoinformatie. Geo - Info: tijdschrift voor geo - informatie Nederland (GIN) 5(5) (2008)

22. de Vries, W.T.: A review of the political nature of ICT in $\mathrm{G} 2 \mathrm{G}$ integration - based on 3 cases from the geoICT domain. In: Proceedings of the 2008 International Conference on Digital Government Research, May 18-21. ACM International Conference Proceeding Series, vol. 289, pp. 124-131. Digital Government Society of North America, Montreal, Canada (2008) ISBN:978-1-60558-099-9

23. de Vries, W.T.: Unity in Diversity: An Analysis of Inter-governmental Cooperation in the Field of geoICT. In: Wimmer, M.A., Scholl, H.J., Ferro, E. (eds.) EGOV 2008. LNCS, vol. 5184, pp. 172-183. Springer, Heidelberg (2008)

24. Miscione, G., Staring, K., Georgiadou, P.Y.: A federative view for information infrastructures in developing contexts. In: Byrne, E., Nicholson, B., Salem, F. (eds.) Proceedings of the 10th International Conference on Social Implications of Computers in Developing Countries: Assessing the Contribution of ICT to Development Goals, Dubai, United Arab Emirates, May 26-28, pp. 209-221. Dubai School of Government, Dubai (2009)

25. Lee, T.W.: Using qualitative methods in organizational research. Sage, Thousand Oaks (1999)

26. Lewins, A., Silver, C.: Using Software in Qualitative Research: A Step-by-Step Guide. Sage Publications, London (2007) 